\title{
Influence of inerter on natural frequencies of vibration systems
}

\author{
Michael Z. Q. Chen ${ }^{\mathrm{a}, *}$, Yinlong $\mathrm{Hu}^{\mathrm{b}}$, Lixi Huang ${ }^{\mathrm{a}}$, Guanrong Chen ${ }^{\mathrm{c}}$ \\ ${ }^{a}$ Department of Mechanical Engineering, The University of Hong Kong, Hong Kong \\ ${ }^{b}$ School of Automation, Nanjing University of Science and Technology, Nanjing, China \\ ${ }^{c}$ Department of Electronic Engineering, City University of Hong Kong, Hong Kong
}

\begin{abstract}
This paper investigates the influence of inerter on the natural frequencies of vibration systems. First of all, the natural frequencies of a single-degree-of-freedom (SDOF) system and a two-degree-of-freedom (TDOF) system are derived algebraically and the fact that the inerter can reduce the natural frequencies of these systems is demonstrated. Then, to further investigate the influence of inerter in a general vibration system, a multi-degree-of-freedom system (MDOF) is considered. Sensitivity analysis is performed on the natural frequencies and mode shapes to demonstrate that the natural frequencies of the MDOF system can always be reduced by increasing the inertance of any inerter. The condition for a general MDOF system of which the natural frequencies can be reduced by an inerter is also derived. Finally, The influence of inerter position on the natural frequencies is investigated and the efficiency of inerter in reducing the largest natural frequencies is verified by simulating a six-degree-of-freedom system, where a reduction of more than $47 \%$ is obtained by employing only five inerters.
\end{abstract}

Keywords: Inerter, natural frequency, vibration analysis.

\section{Introduction}

Inerter is a recently proposed concept and a device with the property that the applied force at its two terminals is proportional to the relative acceleration between them $[1,2]$. As a new passive mechanical element, the performance benefits of using inerters in various mechanical systems have been well demonstrated [2]. In [3], improvements of about $10 \%$ or greater in performance benefits were obtained by incorporating an inerter in vehicle suspension systems after comparing six simple suspension struts. An analytical solution was given in [4] to confirm the performance benefits reported in [3] and a new simple strut containing an inerter was also introduced (S5 in [4]). Inerter has also rekindled interest in passive network synthesis $[5,6,7,8,9,10]$. In particular, five different mechanical networks, which cover all admittances that can be realized with one damper, one inerter and an arbitrary number of springs, were proposed in [8] and the performances of these five networks as vehicle suspensions were studied in [11]. Other than these fix-structured mechanical networks, an approach to optimizing all passive transfer functions (positive-real admittances) with fixed orders by the Linear Matrix Inequalities method was introduced in [12], which

\footnotetext{
${ }^{*}$ Corresponding author. Email: mzqchen@hku.hk.
} 
makes it possible to realize more complex mechanical networks employing inerters. In addition, the performance benefits of using inerters in motorcycle steering systems [13, 14], train suspensions $[15,16,17,18]$ and building vibration control [19] were also reported.

Note that among these applications, inerter always appears in some mechanical networks which possess more complex structures than the conventional networks consisting of only springs and dampers. The networks with inerters will surely be better than or at least equal to the conventional networks consisting of only springs and dampers as they can always reduce to the conventional ones when the values of element coefficients (spring stiffness, damping coefficient or inertance) become zero or infinity [11]. It is true that inerter can provide extra flexibility in structure, but the basic functionality of inerter in vibration systems has not yet been clearly understood and demonstrated.

It is well known that in a vibration system, spring can store energy, provide static support and determine the natural frequencies, while viscous damper can dissipate energy, limit the amplitude of oscillation at resonance and slightly decrease the natural frequencies if the damping is small [20]. As shown in [1], inerter can store energy. However, for the other inherent properties of vibration systems such as natural frequencies, the influence of inerter has not been investigated before.

The objective of this paper is to study the fundamental influence of inerter on the natural frequencies of vibration systems. The fact that inerter can reduce the natural frequencies of vibration systems is theoretically demonstrated in this paper and the question that how to efficiently use inerter to reduce the natural frequencies is also addressed. It is well known that natural frequencies are inherent properties of a vibration system, where resonance may occur when the frequency of the excitation is equal to one of the natural frequencies [21]. In practice, it is always desirable to adjust the natural frequencies of a vibration system to avoid or induce resonance where appropriate. For example, for vibration-based self-powered systems [22], the natural frequency of an embedded spring-mass system should be consistent with the environment to obtain maximum vibration power by utilizing resonance, while for the engine mounting systems [23], the natural frequency should be below the engine disturbance frequency of the engine idle speed to avoid excitation of mounting system resonance. The traditional methods to reduce the natural frequencies of an elastic system are either decreasing the elastic stiffness or increasing the mass of the vibration system. However, this may be problematic; for example, the stiffness values of an engine mount that are too low will lead to large static and quasi-static engine displacement and damage of some engine components [23]. It will be shown below that other than these two methods, a parallel-connected inerter can also effectively reduce natural frequencies.

Since the influence of damping on natural frequencies is well known, only the undamped conservative systems are considered for simplicity. The organization of this paper is as follows. In Section 2, Section 3 and Section 4, single-degree-of-freedom (SDOF) system, two-degreeof-freedom (TDOF) system and multi-degree-of-freedom (MDOF) system are investigated, respectively. The influence of the inerter position on the natural frequencies is investigated in Section 5. A simple design procedure is given in Section 6 to demonstrate the efficiency of inerter in reducing the largest natural frequency of a vibration system. Conclusions are drawn in Section 7. 


\section{SDOF system}

A SDOF system with an inerter is shown in Fig. 1. The equation of motion for free vibration of this system is

$$
(m+b) \ddot{x}+k x=0 .
$$

Transformation of the above equation into the standard form for vibration analysis yields

$$
\ddot{x}+\omega_{n}^{2} x=0,
$$

where $\omega_{n}=\sqrt{\frac{k}{m+b}}$ is called the natural frequency of the undamped system.

Proposition 1. The natural frequency $\omega_{n}$ of an SDOF system is a decreasing function of the inertance $b$. Thus, inerter can reduce the natural frequency of an SDOF system.

Remark 1. Note that in [1], one application of inerter is to simulate the mass by connecting a terminal of an inerter to the mechanical ground. Observing (1), one concludes that the inerter with one terminal connected to ground can effectively enlarge the mass which is connected at the other terminal.

\section{TDOF system}

To investigate the general influence of inerter on the natural frequencies of a vibration system, a TDOF system, shown in Fig. 2, is investigated in this section.

The equations of motion for free vibration of this system are

$$
\begin{aligned}
m_{1} \ddot{x}_{1}+k_{1}\left(x_{1}-x_{2}\right)+b_{1}\left(\ddot{x}_{1}-\ddot{x}_{2}\right) & =0, \\
m_{2} \ddot{x}_{2}-k_{1}\left(x_{1}-x_{2}\right)-b_{1}\left(\ddot{x}_{1}-\ddot{x}_{2}\right)+k_{2} x_{2}+b_{2} \ddot{x}_{2} & =0,
\end{aligned}
$$

or, in a compact form,

$$
\mathbf{M} \ddot{\mathbf{x}}+\mathbf{K x}=0,
$$

where $\mathbf{M}$ is called the inertia matrix and $\mathbf{K}$ the stiffness matrix [21], and

$$
\mathbf{M}=\left[\begin{array}{cc}
m_{1}+b_{1} & -b_{1} \\
-b_{1} & m_{2}+b_{1}+b_{2}
\end{array}\right], \quad \mathbf{K}=\left[\begin{array}{cc}
k_{1} & -k_{1} \\
-k_{1} & k_{1}+k_{2}
\end{array}\right]
$$

Note that the inertances $b_{1}$ and $b_{2}$ only exist in the inertia matrix $\mathbf{M}$, but the positions of $b_{1}$ and $b_{2}$ are different as $b_{1}$ exists in all the elements of $\mathbf{M}$ while $b_{2}$ only appears in the last element of $\mathbf{M}$. Since one terminal of $b_{2}$ is connected to the ground, $b_{2}$ effectively enlarges the mass $m_{2}$, which is consistent with the conclusion made in Remark 1 .

The two natural frequencies can be obtained by solving the characteristic equation [21]

$$
\begin{aligned}
\Delta(\omega)= & \left|\mathbf{K}-\mathbf{M} \omega^{2}\right| \\
= & \left(m_{1} m_{2}+m_{1}\left(b_{1}+b_{2}\right)+m_{2} b_{1}+b_{1} b_{2}\right) \omega^{4}-\left(\left(m_{1}+m_{2}\right) k_{1}+m_{1} k_{2}+k_{1} b_{2}+b_{1} k_{2}\right) \omega^{2} \\
& +k_{1} k_{2}=0,
\end{aligned}
$$


which yields

$$
\begin{aligned}
& \omega_{n 1}=\sqrt{\frac{k_{1} k_{2}\left(f_{1}+f_{2}-\sqrt{\left(f_{1}-f_{2}\right)^{2}+4 d_{0}}\right)}{2\left(f_{1} f_{2}-d_{0}\right)}}, \\
& \omega_{n 2}=\sqrt{\frac{k_{1} k_{2}\left(f_{1}+f_{2}+\sqrt{\left(f_{1}-f_{2}\right)^{2}+4 d_{0}}\right)}{2\left(f_{1} f_{2}-d_{0}\right)}},
\end{aligned}
$$

where $f_{1}=\left(m_{1}+m_{2}+b_{2}\right) k_{1}, f_{2}=\left(m_{1}+b_{1}\right) k_{2}$, and $d_{0}=k_{1} k_{2} m_{1}^{2}$.

Proposition 2. For a TDOF system with two inerters, both natural frequencies $\omega_{n 1}$ and $\omega_{n 2}$ are decreasing functions of the inertance $b_{1}$ and $b_{2}$.

Proof. See Appendix A.

\section{MDOF system}

From the previous two sections, one sees that inerter can reduce the natural frequencies of both SDOF and TDOF systems. To find out whether this holds for any vibration system, a general MDOF system, shown in Fig. 3, is investigated in this section.

The equations of motion of the MDOF system shown in Fig. 3 are

$$
\mathbf{M} \ddot{\mathbf{x}}+\mathbf{K x}=0,
$$

where $\mathbf{x}=\left[x_{1}, x_{2}, \ldots, x_{n}\right]^{T}$, and

$$
\begin{aligned}
& \mathbf{M}=\left[\begin{array}{cccc}
m_{1}+b_{1} & -b_{1} & & \\
-b_{1} & m_{2}+b_{1}+b_{2} & -b_{2} & \\
& \ddots & \ddots & \ddots \\
& & -b_{n-1} & m_{n}+b_{n-1}+b_{n}
\end{array}\right], \\
& \mathbf{K}=\left[\begin{array}{cccc}
k_{1} & -k_{1} & & \\
-k_{1} & k_{1}+k_{2} & -k_{2} & \\
& \ddots & \ddots & \ddots \\
& & -k_{n-1} & k_{n-1}+k_{n}
\end{array}\right] \text {. }
\end{aligned}
$$

It is well known that the free vibration of the MDOF system can be described by the eigenvalue problem as follows [20, 27]

$$
\left(\mathbf{K}-\mathbf{M} \lambda_{j}\right) \varphi_{j}=\mathbf{0}
$$

where $j=1, \ldots, n, \omega_{n j}=\sqrt{\lambda_{j}}$ are the natural frequencies of this system, and $\boldsymbol{\varphi}_{j}$ is the $j$ th mode shape corresponding to natural frequency $\omega_{n j}$ and is normalized to be unit-mass mode shapes, i.e., $\boldsymbol{\varphi}_{j}{ }^{T} \mathrm{M} \boldsymbol{\varphi}_{\boldsymbol{j}}=1$.

Sensitivity analysis is performed on the eigenvalues and eigenvectors with respect to each inertance and the following proposition is derived. 
Proposition 3. Consider the MDOF system shown in Fig. 3. For an arbitrary eigenvalue $\lambda_{j}, j=1, \ldots, n$, and an arbitrary inertance $b_{i}, i=1, \ldots, n$, the following equations hold:

$$
\begin{aligned}
\frac{\partial \lambda_{j}}{\partial b_{i}} & =-\lambda_{j} \Phi_{i j}, \\
\frac{\partial \Phi_{i j}}{\partial b_{i}} & =2 \Phi_{i j}\left(-\frac{1}{2} \Phi_{i j}+\sum_{l=1, l \neq j}^{n} \frac{\lambda_{j}}{\lambda_{l}-\lambda_{j}} \Phi_{i l}\right), \\
\frac{\partial^{2} \lambda_{j}}{\partial b_{i}^{2}} & =2 \lambda_{j} \Phi_{i j}\left(\Phi_{i j}-\sum_{l=1, l \neq j}^{n} \frac{\lambda_{j}}{\lambda_{l}-\lambda_{j}} \Phi_{i l}\right),
\end{aligned}
$$

where $\Phi_{i j}, j=1, \ldots, n$, is defined as

$$
\Phi_{i j}=\varphi_{j}{ }^{T} \frac{\partial \mathrm{M}}{\partial b_{i}} \boldsymbol{\varphi}_{j}= \begin{cases}\left(\varphi_{j}^{(i)}-\varphi_{j}^{(i+1)}\right)^{2}, & i \neq n \\ \left(\varphi_{j}^{(n)}\right)^{2}, & i=n\end{cases}
$$

Proof. See Appendix B.

It is clearly shown in (6) that

$$
\frac{\partial \lambda_{j}}{\partial b_{i}} \leq 0,
$$

and the equality is achieved if $\varphi_{j}{ }^{(i)}=\varphi_{j}{ }^{(i+1)}$ for $i \neq n$ or $\varphi_{j}{ }^{(n)}=0$ for $i=n$. Since $j$ and $i$ are arbitrarily selected, (6) holds for any natural frequency with respect to any inertance $b_{i}$, which means that the natural frequencies of the MDOF system can always be reduced by increasing the inertance of any inerter.

Note that for a discrete vibration system, $\lambda_{j}>0, j=1, \ldots, n$ always holds (if $\lambda_{j}=0$, the vibration system reduces to a lower degree of freedom system), then the necessary and sufficient condition for $\frac{\partial \lambda_{j}}{\partial b_{i}} \leq 0$ is

$$
\frac{\partial \mathbf{M}}{\partial b_{i}} \geq 0 .
$$

Thus, one obtains the following proposition:

Proposition 4. 1. The natural frequencies of the MDOF system shown in Fig. 3 can always be reduced by increasing the inertance of any inerter.

2. The natural frequencies of any MDOF system can be reduced by an inerter if the inertial matrix satisfies (9).

Remark 2. The second conclusion in Proposition 4 means that the vibration systems of which the natural frequencies can be reduced by using an inerter are not restricted to the "uni-axial" MDOF system shown in Fig. 3, but any MDOF system satisfying (9), such as full-car suspension systems [3], train suspension systems [16, 17, 18], buildings [19], etc.

Remark 3. Proposition 4 is easy to interpret physically: for a small increment of inertance $\varepsilon_{b_{i}}$ of a particular inerter $b_{i}$, one obtains

$$
\mathbf{M}=\mathbf{M}_{0}+\varepsilon_{b_{i}} \frac{\partial \mathbf{M}}{\partial b_{i}},
$$


where $\mathbf{M}_{\mathbf{0}}$ is the original inertial matrix. Sine $\frac{\partial \mathbf{M}}{\partial b_{i}}$ is positive semidefine, (10) can be interpreted as increasing the mass of the whole system, which will surely result in the reduction of natural frequencies.

Note that from Proposition 4, it seems that any natural frequency of an MDOF system will be reduced if an inerter with a relatively large value of inertance is inserted, since the added inertance can always be viewed as an integration of small increments. However, this is not always true since there exist permutations of two particular natural frequencies if the divergence between two eigenvalues of the original system is not large enough or the increment of inertance $\varepsilon_{b_{i}}$ is not small enough. Fig. 5 shows the permutation of the natural frequencies of a three-degree-of-freedom system. As shown in Fig. 5, if one denotes the eigenvalues in the order of $\lambda_{1} \geq \lambda_{2} \geq \ldots \geq \lambda_{n}$ all the time, the $\lambda_{i}, i=1, \ldots, n$, will always decrease when the inertance increases. Hence, in the following sections, the eigenvalues are always sorted in a descend order unless otherwise stated.

\section{Influence of the inerter position on the natural frequencies}

The fact that inerter can reduce the natural frequencies of any MDOF system satisfying (9) has been demonstrated. However, for an MDOF system such as the "uni-axial" MDOF system shown in Fig. 3, the influence of inerter position on a specific natural frequency is still unknown. In particular, a practical problem in using inerters to reduce system natural frequencies is: for a specific natural frequency such as the largest natural frequency, where is the most efficient position to insert an inerter so that the largest reduction will be achieved? A TDOF system shown in Fig. 2 will be investigated in detail and analytical solutions will be derived for the TDOF system.

Considering (B.4) with $n=2$, one obtains

$$
\begin{aligned}
\frac{\partial \lambda_{j}}{\partial b_{1}} & =-\lambda_{j}\left(\boldsymbol{\varphi}_{j}^{(\mathbf{1})}-\boldsymbol{\varphi}_{j}^{(\mathbf{2})}\right)^{2}, \\
\frac{\partial \lambda_{j}}{\partial b_{2}} & =-\lambda_{j}\left(\boldsymbol{\varphi}_{j}^{(\mathbf{2})}\right)^{2},
\end{aligned}
$$

where $j=1,2$.

For a small increment of inertance, to compare the efficiency of reducing natural frequencies in terms of $b_{1}$ and $b_{2}$, it is equivalent to compare the absolute values of the derivatives in (11) and (12). Then, the following proposition can be derived.

Proposition 5. For a small increment of inertance and for a specific $\lambda_{j}, j=1,2$, it is more efficient to increase $b_{1}$ than $b_{2}$ if

$$
\frac{k_{1}}{2 m_{1}+b_{1}}<\lambda_{j 0}<\frac{k_{1}}{b_{1}}
$$

or

$$
\lambda_{j 0}>\frac{k_{2}}{m_{2}+b_{2}}, \text { or } \lambda_{j 0}<\frac{k_{2}}{m_{2}+b_{2}+2 m_{1}} ;
$$

It is more efficient to increase $b_{2}$ than $b_{1}$ if

$$
\lambda_{j 0}>\frac{k_{1}}{b_{1}}, \text { or } \lambda_{j 0}<\frac{k_{1}}{b_{1}+2 m_{1}},
$$


or

$$
\frac{k_{2}}{m_{2}+b_{2}+2 m_{1}}<\lambda_{j 0}<\frac{k_{2}}{m_{2}+b_{2}} .
$$

where $\lambda_{j 0}, j=1,2$ denote the eigenvalues of the original system.

Proof. See Appendix C.

Note that (13) and (14), (15) and (16) are equivalent, because (C.1) and (C.2) are equivalent. Proposition 5 is only applied to the case that the increment of inertance is small, as it is obtained by comparing the slopes of the tangent lines as shown in Appendix C. If large increments of inertance are allowed for a given system that can be modeled as Fig. 2 and no inerter is employed in the original system, the question that which is more efficient in terms of $b_{1}$ and $b_{2}$ will be investigated as follows.

To answer this question, one needs to check two situations, where $b_{2}=0$ or $b_{1}=0$, respectively. If $b_{2}=0, b_{1}=b$, from (3) and (4), one has

$$
\begin{aligned}
\omega_{n 1} & =\sqrt{\frac{\left(m_{1}+m_{2}\right) k_{1}+m_{1} k_{2}+k_{2} b_{1}-\sqrt{\left(\left(m_{1}+m_{2}\right) k_{1}-m_{1} k_{2}-b_{1} k_{2}\right)^{2}+4 k_{1} k_{2} m_{1}^{2}}}{2\left(m_{1} m_{2}+\left(m_{1}+m_{2}\right) b_{1}\right)}}, \\
\omega_{n 2} & =\sqrt{\frac{\left(m_{1}+m_{2}\right) k_{1}+m_{1} k_{2}+k_{2} b_{1}+\sqrt{\left(\left(m_{1}+m_{2}\right) k_{1}-m_{1} k_{2}-b_{1} k_{2}\right)^{2}+4 k_{1} k_{2} m_{1}^{2}}}{2\left(m_{1} m_{2}+\left(m_{1}+m_{2}\right) b_{1}\right)}} .
\end{aligned}
$$

If $b_{1}=0, b_{2}=b$, one has

$$
\begin{aligned}
\omega_{n 1}^{\prime} & =\sqrt{\frac{\left(m_{1}+m_{2}\right) k_{1}+m_{1} k_{2}+k_{1} b_{2}-\sqrt{\left(\left(m_{1}+m_{2}\right) k_{1}-m_{1} k_{2}+b_{2} k_{1}\right)^{2}+4 k_{1} k_{2} m_{1}^{2}}}{2\left(m_{1} m_{2}+m_{1} b_{2}\right)}}, \\
\omega_{n 2}^{\prime} & =\sqrt{\frac{\left(m_{1}+m_{2}\right) k_{1}+m_{1} k_{2}+k_{1} b_{2}+\sqrt{\left(\left(m_{1}+m_{2}\right) k_{1}-m_{1} k_{2}+b_{2} k_{1}\right)^{2}+4 k_{1} k_{2} m_{1}^{2}}}{2\left(m_{1} m_{2}+m_{1} b_{2}\right)}} .
\end{aligned}
$$

The above question can be answered by comparing $\omega_{n 1}$ and $\omega_{n 2}$ with $\omega_{n 1}^{\prime}$ and $\omega_{n 2}^{\prime}$, respectively. Thus, one has the following proposition.

Proposition 6. Denote

$$
b_{0}=\frac{k_{1} m_{2}\left(2 m_{1} k_{2}-\left(2 m_{1}+m_{2}\right) k_{1}\right)}{\left(k_{2}-k_{1}\right)\left(m_{1} k_{2}-\left(m_{1}+m_{2}\right) k_{1}\right)} .
$$

For the larger natural frequency $\omega_{n 2}$ :

If $k_{2} \leq\left(1+\frac{m_{2}}{m_{1}}\right) k_{1}, b_{1}$ is more efficient than $b_{2}$;

If $k_{2}>\left(1+\frac{m_{2}}{m_{1}}\right) k_{1}, b_{1}$ is more efficient in $\left[0, b_{0}\right] ; b_{2}$ is more efficient in $\left[b_{0},+\infty\right)$.

For the smaller natural frequency $\omega_{n 1}$ :

If $k_{2}>\left(1+\frac{m_{2}}{2 m_{1}}\right) k_{1}, b_{1}$ is more efficient than $b_{2}$;

If $k_{1} \leq k_{2} \leq\left(1+\frac{m_{2}}{2 m_{1}}\right) k_{1}, b_{2}$ is more efficient in $\left[0, b_{0}\right] ; b_{1}$ is more efficient in $\left[b_{0},+\infty\right)$;

If $k_{2}<k_{1}, b_{2}$ is more efficient than $b_{1}$.

Proof. See Appendix D. 
Proposition 6 has addressed four cases, which are $k_{2}>\left(1+m_{2} / m_{1}\right) k_{1},\left(1+m_{2} /\left(2 m_{1}\right)\right) k_{1} \leq$ $k_{2} \leq\left(1+m_{2} / m_{1}\right) k_{1}, k_{1} \leq k_{2}<\left(1+m_{2} /\left(2 m_{1}\right)\right) k_{1}, k_{2} \leq k_{1}$. A numerical example is performed with $m_{1}=m_{2}=100 \mathrm{~kg}, k_{1}=1000 \mathrm{~N} / \mathrm{m}$ and $k_{2}$ chosen as $2500,1800,1300,500 \mathrm{~N} / \mathrm{m}$ corresponding to the four cases in Proposition 6. The results are shown in Fig. 4, where from Fig. 4(a), one sees that in terms of the larger natural frequency, although for small increment of inertance (about $0-250 \mathrm{~kg}$ ) $b_{1}$ is more efficient than $b_{2}$, for large increment of inertance, $b_{2}$ tends to be more efficient than $b_{1}$.

Note that the above discussion is based on TDOF systems. For a general MDOF system, a similar argument as in Proposition 5 can be employed to determine the efficiency of the position of inerter by comparing the absolute values of the derivatives. For example, consider a six-degree-of-freedom system with $m_{i}=100 \mathrm{~kg}, i=1, \ldots, 6$, and $k_{1}=1000 \mathrm{~N} / \mathrm{m}, k_{2}=1000$ $\mathrm{N} / \mathrm{m}, k_{3}=2000 \mathrm{~N} / \mathrm{m}, k_{4}=2000 \mathrm{~N} / \mathrm{m}, k_{5}=3000 \mathrm{~N} / \mathrm{m}, k_{6}=3000 \mathrm{~N} / \mathrm{m}$. The objective is to find out the most efficient position to insert an inerter so that largest reduction of the largest natural frequency will be achieved. By direct calculation, one obtains $\left|\frac{\partial \lambda_{1}}{\partial b_{i}}\right|, i=1, \ldots, 6$ as $2.759 \times 10^{-4}, 0.0134,0.1559,0.8571,1.5999,0.4043$, respectively. Note that $\left|\frac{\partial \lambda_{1}}{\partial b_{5}}\right|$ possesses the largest value. Hence, the position between $m_{5}$ and $m_{6}$ would be the most efficient position to insert an inerter, which is consistent with the simulation shown in Fig. 6. Another method to find the most efficient position is by using Gershgorin's Theorem [24], which shows that the largest absolute row sums is an upper bound of the largest eigenvalue. Hence, an efficient way to reduce the largest natural frequency is to insert the inerter between the mass $m_{j}$ and $m_{j+1}$ or $m_{j-1}$ and $m_{j}$, where the $j$ th absolute row sum of $\mathbf{M}^{-\mathbf{1}} \mathbf{K}$ is the largest absolute row sum of $\mathbf{M}^{-\mathbf{1}} \mathbf{K}$. Taking the same six-degree-of-freedom system as an example, one obtains

$$
\mathbf{M}^{-\mathbf{1}} \mathbf{K}=\left[\begin{array}{cccccc}
10 & -10 & 0 & 0 & 0 & 0 \\
-10 & 20 & -10 & 0 & 0 & 0 \\
0 & -10 & 30 & -20 & 0 & 0 \\
0 & 0 & -20 & 40 & -20 & 0 \\
0 & 0 & 0 & -20 & 50 & -30 \\
0 & 0 & 0 & 0 & -30 & 60
\end{array}\right]
$$

The absolute row sums of $\mathbf{M}^{-\mathbf{1}} \mathbf{K}$ are $20,40,60,80,100$, and 90 . Thus, one concludes that the optimal way is to insert an inerter between $m_{5}$ and $m_{6}$, which is consistent with the simulation shown in Fig. 6 as well.

\section{Design procedure and numerical example}

The problem of reducing the largest natural frequency of a vibration system is considered in this section, where the efficiency of inerter in reducing natural frequencies will be quantitatively shown.

For the largest natural frequency, considering (7) and (8), one obtains

$$
\frac{\partial \Phi_{i j}}{\partial b_{i}} \leq 0 \text {, and } \frac{\partial^{2} \lambda_{j}}{\partial b_{i}^{2}} \geq 0 .
$$

Note that $\Phi_{i j} \geq 0$ and the equality is achieved with $\varphi_{j}^{(i)}=\boldsymbol{\varphi}_{j}^{(i+1)}$ when $i \neq n$, or $\boldsymbol{\varphi}_{j}^{(n)}=0$ when $i=n$, which means that for a specific inerter $b_{i}, i=1, \ldots, n$, the largest natural 
Table 1: Structure Model Parameters.

\begin{tabular}{ll}
\hline Floor masses $(\mathrm{kg})$ & Stiffness coefficients $(\mathrm{kN} / \mathrm{m})$ \\
\hline$m_{1}=5897$ & $k_{1}=19059$ \\
$m_{2}=5897$ & $k_{2}=24954$ \\
$m_{3}=5897$ & $k_{3}=28621$ \\
$m_{4}=5897$ & $k_{4}=29093$ \\
$m_{5}=5897$ & $k_{5}=33732$ \\
$m_{6}=6800$ & $k_{6}=232$ \\
\hline
\end{tabular}

Table 2: Procedures and results.

\begin{tabular}{|c|c|c|c|c|c|c|c|c|}
\hline Steps & \multicolumn{6}{|c|}{ Inertance $(\mathrm{kg})$} & $\omega_{\max }(\mathrm{rad} / \mathrm{s})$ & Percentages \\
\hline 1 st & $b_{4}=5000$ & & & & & & 118.89 & $(11.22 \%)$ \\
\hline 2 th & $b_{4}=5000$ & $b_{2}=5000$ & & & & & 100.19 & $(25.18 \%)$ \\
\hline 3 th & $b_{4}=5000$ & $b_{2}=5000$ & $b_{5}=5000$ & & & & 90.49 & $(32.43 \%)$ \\
\hline 4 th & $b_{4}=5000$ & $b_{2}=5000$ & $b_{5}=5000$ & $b_{3}=3000$ & & & 78.15 & $(41.64 \%)$ \\
\hline 5 th & $b_{4}=5000$ & $b_{2}=5000$ & $b_{5}=5000$ & $b_{3}=3000$ & $b_{1}=1000$ & & 70.95 & $(47.02 \%)$ \\
\hline 6 th & $b_{4}=5000$ & $b_{2}=5000$ & $b_{5}=5000$ & $b_{3}=3000$ & $b_{1}=1000$ & $b_{6}=1 \times 10^{5}$ & 70.91 & $(47.05 \%)$ \\
\hline
\end{tabular}

frequency will always be reduced by increasing the inertance until the two masses connected by inerter $b_{i}$ are rigidly connected.

In what follows, an intuitive and simple approach to lowering the largest natural frequency for a given structure is illustrated by inserting the inerters one by one, where the inerter in each step is placed at the most efficient position. Here, a procedure is presented to reduce the largest natural frequency of a structure discussed in $[25,26]$ with parameters given in Table 1 . Note that the largest natural frequency $\omega_{\max }$ of this structure is $133.91 \mathrm{rad} / \mathrm{s}$. The procedure to reduce $\omega_{\max }$ is shown in Fig. 7 and Table 2.

\section{Procedure description:}

Step 1 Fig. 7(a) shows that $b_{4}$ is the most efficient regarding the original system and for $b_{4}>5000 \mathrm{~kg}, \omega_{\max }$ decreases slightly, hence $b_{4}=5000 \mathrm{~kg}$ is selected;

Step 2 Fig. 7(b) shows that $b_{2}$ is the most efficient regarding the original system and $b_{4}$ and $b_{2}>5000 \mathrm{~kg}, \omega_{\max }$ decreases slightly, hence $b_{2}=5000 \mathrm{~kg}$ is selected;

Step 3-Step 6 Similarly, from Fig. 7(c) to Fig. 7(f), $b_{5}=5000 \mathrm{~kg}, b_{3}=3000 \mathrm{~kg}, b_{1}=1000$ $\mathrm{kg}$ and $b_{6}=1 \times 10^{5} \mathrm{~kg}$ are selected, respectively.

Note that the above-illustrated approach is not optimal as the natural frequencies of a system can always be reduced by enlarging the inertances until the inertial matrix $\mathbf{M}$ became singular, where all the natural frequencies become zero. However, the efficiency of inerter in reducing natural frequencies can be clearly demonstrated by this approach. As shown in Table 2, attenuation about $47.05 \%$ has been obtained. It is worth pointing out that the required inertance for $b_{6}$ is $1 \times 10^{5} \mathrm{~kg}$, which is quite large. However, the reduction of largest natural frequency is only improved by $0.03 \%$. If the cost factor is considered in practice, $b_{6}$ can be omitted. In this way, only five inerters are employed. 


\section{Conclusion}

This paper has investigated the influence of inerter on the natural frequencies of vibration systems. By algebraically deriving the natural frequencies of a SDOF system and a TDOF system, the fact that inerter can reduce the natural frequencies of these systems has been clearly demonstrated. To reveal the influence of inerter on the natural frequencies of a general system, an MDOF system has been considered. Sensitivity analysis has been performed on the natural frequencies and mode shapes to demonstrate that any increment of the inertance of any inerter in an MDOF system results in a reduction of the natural frequencies. To that end, the effectiveness of inerter in reducing natural frequencies of a general vibration system has been clearly demonstrated. Finally, the influence of the inerter position has been investigated and a simple design procedure has been proposed to verify the efficiency of inerter in reducing the largest natural frequencies of vibration systems. The simulation result has shown that more than $47 \%$ reduction can be obtained with only five inerters employed in a six-degree-of-freedom vibration system.

\section{Acknowledgements}

This work is supported by Hong Kong University Committee on Research and Conference Grants under Grant 201111159110, National Natural Science Foundation of China under Grant 61004093, National Key Basic Research Scheme of China (973 Program) under Grant 2012CB720202, and the Hong Kong Research Grants Council under the GRF Grant CityU1109/12.

\section{Appendix A. Proof of Proposition 2}

The monotonicity of $\omega_{n 1}$ and $\omega_{n 2}$ can be proven by checking the signs of the first-order derivatives of $\omega_{n 1}^{2}$ and $\omega_{n 2}^{2}$ in terms of $f_{1}$ and $f_{2}$, respectively.

$$
\begin{aligned}
\frac{\partial \omega_{n 1}^{2}}{\partial f_{1}} & =-\frac{k_{1} k_{2}\left(q_{1}-q_{2}\right)}{2\left(d_{0}-f_{1} f_{2}\right)^{2} \sqrt{\left(f_{1}-f_{2}\right)^{2}+4 d_{0}}}, \\
\frac{\partial \omega_{n 2}^{2}}{\partial f_{1}} & =-\frac{k_{1} k_{2}\left(q_{1}+q_{2}\right)}{2\left(d_{0}-f_{1} f_{2}\right)^{2} \sqrt{\left(f_{1}-f_{2}\right)^{2}+4 d_{0}}},
\end{aligned}
$$

where $q_{1}=\left(d_{0}+f_{2}^{2}\right) \sqrt{\left(f_{1}-f_{2}\right)^{2}+4 d_{0}}$ and $q_{2}=f_{1}\left(d_{0}-f_{2}^{2}\right)+3 f_{2} d_{0}+f_{2}^{3}$.

Note that $q_{1}>0$ and

$$
q_{1}^{2}-q_{2}^{2}=4 d_{0} f_{2}^{2}\left(f_{1}-d_{0} / f_{2}\right)^{2}
$$

so one obtains $\left|q_{1}\right|>\left|q_{2}\right|$, which implies $\frac{\partial \omega_{n 1}^{2}}{\partial f_{1}}<0$ and $\frac{\partial \omega_{n 2}^{2}}{\partial f_{1}}<0$, that is, both $\omega_{n 1}$ and $\omega_{n 2}$ are decreasing functions of inertance $b_{2}$.

Similarly,

$$
\begin{aligned}
\frac{\partial \omega_{n 1}^{2}}{\partial f_{2}} & =-\frac{k_{1} k_{2}\left(q_{3}-q_{4}\right)}{2\left(d_{0}-f_{1} f_{2}\right)^{2} \sqrt{\left(f_{1}-f_{2}\right)^{2}+4 d_{0}}}, \\
\frac{\partial \omega_{n 2}^{2}}{\partial f_{2}} & =-\frac{k_{1} k_{2}\left(q_{3}+q_{4}\right)}{2\left(d_{0}-f_{1} f_{2}\right)^{2} \sqrt{\left(f_{1}-f_{2}\right)^{2}+4 d_{0}}}
\end{aligned}
$$


where $q_{3}=\left(d_{0}+f_{1}^{2}\right) \sqrt{\left(f_{1}-f_{2}\right)^{2}+4 d_{0}}$ and $q_{4}=f_{2}\left(d_{0}-f_{1}^{2}\right)+3 f_{1} d_{0}+f_{1}^{3}$.

Since $q_{3}>0$ and $q_{3}^{2}-q_{4}^{2}=4 d_{0} f_{1}^{2}\left(f_{2}-d_{0} / f_{1}\right)^{2}>0$, one has $\left|q_{3}\right|>\left|q_{4}\right|, \frac{\partial \omega_{n 1}^{2}}{\partial f_{2}}<0$, and $\frac{\partial \omega_{n 2}^{2}}{\partial f_{2}}<0$, that is, both $\omega_{n 1}$ and $\omega_{n 2}$ are decreasing functions of inertance $b_{1}$.

\section{Appendix B. Proof of Proposition 3}

The proof is inspired by the sensitivity analysis on natural frequencies (eigenvalues) and model shapes (eigenvectors) with respect to structure parameters in [27, 28, 29].

Sensitivity on natural frequencies:

Considering the influence of the $i$ th inertance $b_{i}$ on the $j$ th natural frequency $\omega_{n j}$, the derivative of (5) with respect to $b_{i}$ is

$$
\left(\frac{\partial \mathbf{K}}{\partial b_{i}}-\frac{\partial \lambda_{j}}{\partial b_{i}} \mathbf{M}-\lambda_{j} \frac{\partial \mathbf{M}}{\partial b_{i}}\right) \boldsymbol{\varphi}_{j}+\left(\mathbf{K}-\lambda_{j} \mathbf{M}\right) \frac{\partial \boldsymbol{\varphi}_{j}}{\partial b_{i}}=0 .
$$

Premultiplying both sides of (B.1) by $\varphi_{j}{ }^{T}$ and considering the relations that $\frac{\partial \mathbf{K}}{\partial b_{i}}=0(\mathbf{K}$ is independent of $\left.b_{i}\right), \varphi_{j}{ }^{T}\left(\mathbf{K}-\lambda_{j} \mathbf{M}\right)=0$, and $\boldsymbol{\varphi}_{j}{ }^{T} \mathbf{M} \boldsymbol{\varphi}_{j}=1$, one obtains

$$
\frac{\partial \lambda_{j}}{\partial b_{i}}=-\lambda_{j} \boldsymbol{\varphi}_{j}^{T} \frac{\partial \mathbf{M}}{\partial b_{i}} \boldsymbol{\varphi}_{j}
$$

Note that

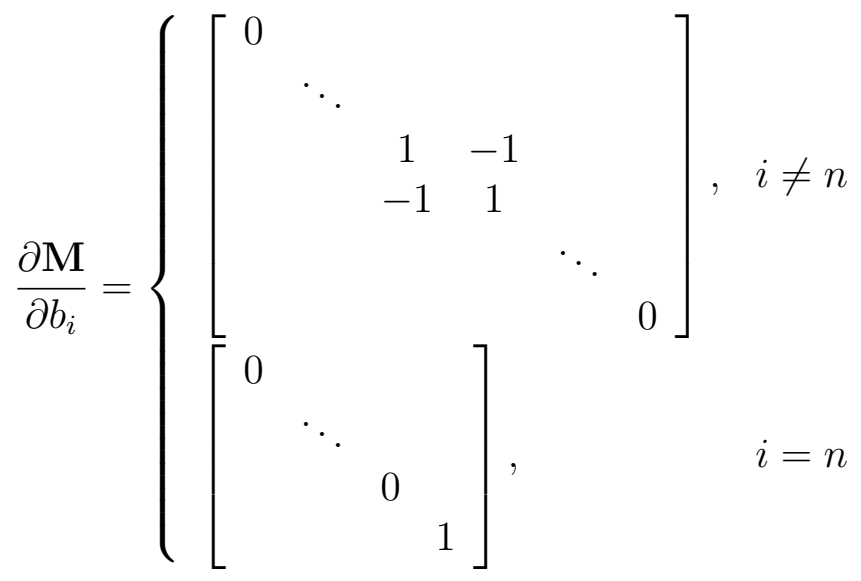

where the nonzero elements for the case $i \neq n$ locate on the $i$ th, $i+1$ th rows and $i$ th, $i+1$ th columns.

Thus, one obtains

$$
\frac{\partial \lambda_{j}}{\partial b_{i}}= \begin{cases}-\lambda_{j}\left(\boldsymbol{\varphi}_{j}^{(i)}-\boldsymbol{\varphi}_{j}^{(i+1)}\right)^{2}, & i \neq n \\ -\lambda_{j}\left(\boldsymbol{\varphi}_{j}^{(n)}\right)^{2}, & i=n\end{cases}
$$

where $\boldsymbol{\varphi}_{j}{ }^{(i)}, i=1, \ldots, n$, denotes the $i$ th element of $\boldsymbol{\varphi}_{j}$.

Denoting

$$
\Phi_{i j}=\varphi_{j}{ }^{T} \frac{\partial \mathrm{M}}{\partial b_{i}} \varphi_{j}= \begin{cases}\left(\varphi_{j}^{(i)}-\varphi_{j}^{(i+1)}\right)^{2}, & i \neq n \\ \left(\varphi_{j}^{(n)}\right)^{2}, & i=n\end{cases}
$$


where $j=1, \ldots, n$, one obtains $(6)$.

Sensitivity on mode shapes:

The method in [27] is adopted. Note that the total behavior of the vibration system can be defined by using the $n$ independent eigenvectors (mode shapes) in an $n$-dimensional vector space. Hence, the derivative of the $j$ th mode shape can be defined by using the $n$ independent eigenvectors as follows:

$$
\frac{\partial \boldsymbol{\varphi}_{j}}{\partial b_{i}}=\sum_{l=1}^{n} \alpha_{l} \boldsymbol{\varphi}_{l}
$$

where $\alpha_{l}, l=1, \ldots, n$ is the weight of the $l$ th mode shape to be determined.

Two cases exist for $\alpha_{l}$. For the first case, that is, $l \neq j$, premultiply $\varphi_{l}^{T}$ on both sides of (B.1). Since mode shapes are orthogonal, it can be shown that $\varphi_{l}^{T} \mathbf{M} \varphi_{j}=0$, if $l \neq j$, and $\varphi_{j}^{T} \mathbf{M} \varphi_{j}=1 ; \varphi_{l}^{T} \mathbf{K}=\lambda_{l} \varphi_{l}^{T} \mathbf{M}$. Then, one obtains

$$
\alpha_{l}=\frac{\lambda_{j}}{\lambda_{l}-\lambda_{j}} \varphi_{l}^{T} \frac{\partial \mathbf{M}}{\partial b_{i}} \boldsymbol{\varphi}_{j} .
$$

Furthermore, considering (B.3), one obtains

$$
\alpha_{l}=\frac{\lambda_{j}}{\lambda_{l}-\lambda_{j}} \Phi_{i l} .
$$

For the second case where $l=j$, since the mode shapes have been normalized to unitmasses, that is

$$
\boldsymbol{\varphi}_{j}^{T} \mathbf{M} \boldsymbol{\varphi}_{j}=1,
$$

where $j=1, \ldots, n$. Taking the derivative of (B.7) with respect to $b_{i}$ results in

$$
\frac{\partial \boldsymbol{\varphi}_{j}{ }^{T}}{\partial b_{i}} \mathbf{M} \boldsymbol{\varphi}_{j}+\boldsymbol{\varphi}_{j}{ }^{T}\left(\frac{\partial \mathbf{M}}{\partial b_{i}} \boldsymbol{\varphi}_{j}+\mathbf{M} \frac{\partial \boldsymbol{\varphi}_{j}}{\partial b_{i}}\right)=0 .
$$

Considering the symmetry property of the inertial matrix, one obtains

$$
2 \boldsymbol{\varphi}_{j}{ }^{T} \mathbf{M} \frac{\partial \boldsymbol{\varphi}_{j}}{\partial b_{i}}=-\boldsymbol{\varphi}_{j}{ }^{T} \frac{\partial \mathbf{M}}{\partial b_{i}} \boldsymbol{\varphi}_{j} .
$$

Substituting (B.5) into (B.8), one obtains

$$
\alpha_{j}=-\frac{1}{2} \boldsymbol{\varphi}_{j}^{T} \frac{\partial \mathbf{M}}{\partial b_{i}} \boldsymbol{\varphi}_{j}=-\frac{1}{2} \Phi_{i j} .
$$

Substituting (B.9) and (B.6) into (B.5), one obtains

$$
\frac{\partial \boldsymbol{\varphi}_{j}}{\partial b_{i}}=-\frac{1}{2} \Phi_{i j} \boldsymbol{\varphi}_{j}+\sum_{l=1, l \neq j}^{n} \frac{\lambda_{j}}{\lambda_{l}-\lambda_{j}} \Phi_{i l} \boldsymbol{\varphi}_{l} .
$$

Considering the $i$ th and $(i+1)$ th elements of $\frac{\partial \varphi_{j}}{\partial b_{i}}$ if $i \neq n$, or $n$th element if $i=n$, and knowing that

$$
\frac{\partial \Phi_{i j}}{\partial b_{i}}= \begin{cases}2\left(\boldsymbol{\varphi}_{j}^{(i)}-\boldsymbol{\varphi}_{j}^{(i+1)}\right) \frac{\partial\left(\varphi_{j}^{(i)}-\varphi_{j}^{(i+1)}\right)}{\partial b_{i}}, & i \neq n \\ 2\left(\boldsymbol{\varphi}_{j}^{(n)}\right) \frac{\partial\left(\varphi_{j}^{(n)}\right)}{\partial b_{i}}, & i=n\end{cases}
$$


one obtains (7).

Since

$$
\frac{\partial^{2} \lambda_{j}}{\partial b_{i}^{2}}=-\frac{\partial \lambda_{j}}{\partial b_{i}} \Phi_{k}-\lambda_{j} \frac{\partial \Phi_{i j}}{\partial b_{i}},
$$

one obtains (8) by substituting (6) and (7) into (B.11).

\section{Appendix C. Proof of Proposition 5}

Considering (5), one obtains

$$
\begin{aligned}
\boldsymbol{\varphi}_{\boldsymbol{j}}^{(1)}-\boldsymbol{\varphi}_{\boldsymbol{j}}{ }^{(2)} & =\frac{\lambda_{j} m_{1}}{k_{1}-\lambda_{j}\left(m_{1}+b_{1}\right)} \boldsymbol{\varphi}_{\boldsymbol{j}}^{(2)}, \\
& =\frac{k_{2}-\lambda_{j}\left(m_{1}+m_{2}+b_{2}\right)}{\lambda_{j} m_{1}} \boldsymbol{\varphi}_{\boldsymbol{j}}^{(2)},
\end{aligned}
$$

where $j=1,2$, and (C.1) is obtained by checking the first row of (5) and (C.2) is obtained by summing the first and second rows of $(5)$.

Note that

$$
\left|\frac{\partial \lambda_{j}}{\partial b_{1}}\right|-\left|\frac{\partial \lambda_{j}}{\partial b_{2}}\right|=\lambda_{j}\left(\left(\boldsymbol{\varphi}_{j}{ }^{(1)}-\boldsymbol{\varphi}_{j}{ }^{(2)}\right)^{2}-\left(\boldsymbol{\varphi}_{j}{ }^{(2)}\right)^{2}\right) .
$$

Substituting (C.1) and (C.2), separately, one obtains the conditions in Proposition 5.

\section{Appendix D. Proof of Proposition 6}

Denote $b_{1}=b_{2}=b$,

$$
\begin{aligned}
& d_{1}=2\left(m_{1} m_{2}+m_{1} b\right) \\
& d_{2}=2\left(m_{1} m_{2}+\left(m_{1}+m_{2}\right) b\right) \\
& d_{3}=\left(m_{1}+m_{2}\right) k_{1}+m_{1} k_{2}+k_{2} b \\
& d_{4}=\left(m_{1}+m_{2}\right) k_{1}+m_{1} k_{2}+k_{1} b, \\
& d_{5}=\sqrt{\left(b k_{2}+m_{1} k_{2}-\left(m_{1}+m_{2}\right) k_{1}\right)^{2}+4 k_{1} k_{2} m_{1}^{2}}, \\
& d_{6}=\sqrt{\left(b k_{1}-m_{1} k_{2}+\left(m_{1}+m_{2}\right) k_{1}\right)^{2}+4 k_{1} k_{2} m_{1}^{2}},
\end{aligned}
$$

and

$$
\begin{aligned}
& F_{1}(b)=\omega_{n 1}^{2}-\omega_{n 1}^{\prime 2}=\frac{d_{1} d_{3}-d_{2} d_{4}-d_{1} d_{5}+d_{2} d_{6}}{d_{1} d_{2}} \\
& F_{2}(b)=\omega_{n 2}^{2}-\omega_{n 2}^{\prime 2}=\frac{d_{1} d_{3}-d_{2} d_{4}+d_{1} d_{5}-d_{2} d_{6}}{d_{1} d_{2}}
\end{aligned}
$$

Also denote

$$
b_{0}=\frac{k_{1} m_{2}\left(2 m_{1} k_{2}-\left(2 m_{1}+m_{2}\right) k_{1}\right)}{\left(k_{2}-k_{1}\right)\left(m_{1} k_{2}-\left(m_{1}+m_{2}\right) k_{1}\right)} .
$$

By direct calculation, it can be easily verified that both $F_{1}(b)=0$ and $F_{2}(b)=0$ have solutions at 0 and $b_{0}$. However, note that $F_{1}(b)$ and $F_{2}(b)$ cannot be zero at the same 
time if $b \neq 0$, thus $F_{1}\left(b_{0}\right)=0$ and $F_{2}\left(b_{0}\right)=0$ cannot hold simultaneously. Particularly, since $b>0$, one is more interested in the cases that $k_{2} \in\left[k_{1},\left(1+m_{2} /\left(2 m_{1}\right)\right) k_{1}\right]$ and $k_{2} \in$ $\left[\left(1+m_{2} / m_{2}\right) k_{1}, \infty\right)$, where $b_{0} \geq 0$.

Next, it is shown that the positive value of $b_{0}$ in $k_{2} \in\left[\left(1+m_{2} / m_{2}\right) k_{1}, \infty\right)$ belongs to $F_{2}(b)=0$ and the other one belongs to $F_{1}(b)=0$. Denote

$$
\begin{aligned}
& \Delta_{2}=m_{1} k_{2}-\left(m_{1}+m_{2}\right) k_{1}, \\
& \Delta_{1}^{2}=\Delta_{2}^{2}+4 k_{1} k_{2} m_{1}^{2} .
\end{aligned}
$$

Then

$$
\begin{aligned}
& d_{5}=\sqrt{b k_{2}^{2}+2 \Delta_{2} k_{2} b+\Delta_{1}^{2}}=k_{2} b+\Delta_{2}+\frac{2 k_{1} m_{1}^{2}}{b}+O\left(\frac{1}{b^{2}}\right), \\
& d_{6}=\sqrt{b k_{1}^{2}-2 \Delta_{2} k_{1} b+\Delta_{1}^{2}}=k_{1} b-\Delta_{2}+\frac{2 k_{2} m_{1}^{2}}{b}+O\left(\frac{1}{b^{2}}\right) .
\end{aligned}
$$

Hence, one has

$$
\begin{aligned}
& F_{2}(b)=\frac{d_{1} d_{3}-d_{2} d_{4}+d_{1} d_{5}-d_{2} d_{6}}{d_{1} d_{2}} \\
= & \frac{\Delta_{2}\left(4 b^{2}+4\left(m_{1}+m_{2}\right) b+4 m_{1} m_{2}\right)-4 m_{1}\left(m_{2} k_{1}-m_{1}\left(k_{1} m_{1}-k_{2}\left(m_{1}+m_{2}\right)\right)\right)+O\left(\frac{1}{b}\right)}{d_{1} d_{2}} .
\end{aligned}
$$

Note that if $\Delta_{2}<0$ and $k_{2}>k_{1}$, or $k_{1}<k_{2}<\left(1+m_{2} / m_{1}\right) k_{1}, F_{2}(b)$ is always negative by omitting the higher-order item $O\left(\frac{1}{b}\right)$. This indicates that if $k_{2}<\left(1+m_{2} / m_{1}\right) k_{1}$, then $F_{2}(b)=0$ only has the trivial solution 0 , while if $k_{2} \geq\left(1+m_{2} / m_{1}\right) k_{1}$, then $F_{2}(b)=0$ has solutions at 0 and $b_{0}$. Consequently, if $k_{2}<\left(1+m_{2} / m_{1}\right) k_{1}$, then $F_{1}(b)=0$ has roots at 0 and $b_{0}$, while if $k_{2} \geq\left(1+m_{2} / m_{1}\right) k_{1}$, then $F_{1}(b)=0$ only has a trivial solution 0 .

Besides, since

$$
\begin{aligned}
F_{1}(b) & =\frac{d_{1} d_{3}-d_{2} d_{4}-d_{1} d_{5}+d_{2} d_{6}}{d_{1} d_{2}}, \\
& =\frac{4 m_{1}\left(m_{1}+m_{2}\right)\left(k_{1}-k_{2}\right) b-4 m_{1}\left(m_{1}^{2}\left(k_{1}-k_{2}\right)-m_{2} k_{1}\left(m_{1}+m_{2}\right)\right)-O\left(\frac{1}{b}\right)}{d_{1} d_{2}},
\end{aligned}
$$

by the relationship of the coefficients and the roots of $F_{1}(b)$ and $F_{2}(b)$, one has:

If $k_{2}>\left(1+m_{2} / m_{1}\right) k_{1}, F_{1}(b) \leq 0$ and $F_{2}(b) \leq 0$ for $b \in\left[0, b_{0}\right], F_{2}(b)>0$ for $b \in\left(b_{0}, \infty\right)$;

If $\left(1+m_{2} /\left(2 m_{1}\right)\right) k_{1} \leq k_{2} \leq\left(1+m_{2} / m_{1}\right) k_{1}, F_{1}(b)<0$ and $F_{2}(b)<0$;

If $k_{1} \leq k_{2}<\left(1+m_{2} /\left(2 m_{1}\right)\right) k_{1}, F_{1}(b) \geq 0$ for $b \in\left[0, b_{0}\right], F_{1}(b)<0$ for $b \in\left(b_{0}, \infty\right)$, and $F_{2}(b)<0$

If $k_{2}<k_{1}, F_{1}(b)>0$ and $F_{2}(b)<0$.

Thus, Proposition 6 and the four cases shown in Fig. 4 haven been proved.

\section{References}

[1] M.C. Smith, Synthesis of mechanical networks: The inerter, IEEE Transactions on Automatic Control 47 (10) (2002) 1648-1662. 
[2] M.Z.Q. Chen, C. Papageorgiou, F. Scheibe, F.C. Wang, M.C. Smith, The missing mechanical circuit element, IEEE Circuits and Systems Magazine 9 (1) (2009) 10-26.

[3] M.C. Smith, F.C. Wang, Performance benefits in passive vehicle suspensions employing inerters, Vehicle System Dynamics 42 (4) (2004) 235-257.

[4] F. Scheibe, M.C. Smith, Analytical solutions for optimal ride comfort and tyre grip for passive vehicle suspensions, Vehicle System Dynamics 47 (10) (2009) 1229-1252.

[5] M.Z.Q. Chen, Passive Network Synthesis of Restricted Complexity, Ph.D. Thesis, Cambridge University Engineering Department, U.K., 2007.

[6] M.Z.Q. Chen, M. C. Smith, Electrical and mechanical passive network synthesis, in Recent Advances in Learning and Control, New York: Springer-Verlag, 371 (2008), pp. $35-50$.

[7] M.Z.Q. Chen, M.C. Smith, A note on tests for positive-real functions, IEEE Transactions on Automatic Control 54 (2) (2009) 390-393.

[8] M.Z.Q. Chen, M.C. Smith, Restricted complexity network realizations for passive mechanical control, IEEE Transactions on Automatic Control 54 (10) (2009) 2290-2301.

[9] M.Z.Q. Chen, K. Wang, Z. Shu, C. Li, Realizations of a special class of admittances with strictly lower complexity than canonical forms, IEEE Transactions on Circuits and Systems-I: Regular Papers, in press. DOI:10.1109/TCSI.2013.2245471

[10] M.Z.Q. Chen, K. Wang, Y. Zou, J. Lam, Realization of a special class of admittances with one damper and one inerter for mechanical control, IEEE Transactions on Automatic Control 58 (7) (2013) 1841-1846.

[11] M.Z.Q. Chen, Y. Hu, B. Du, Suspension performance with one damper and one inerter, Proceedings of the 24th Chinese Control and Decision Conference (CCDC), Tainyuan, China, 2012, pp. 3551-3556.

[12] C. Papageorgiou, M.C. Smith, Positive real synthesis using matrix inequalities for mechanical networks: application to vehicle suspension, IEEE Transactions on Control Systems Technology 14 (3) (2006) 423-435.

[13] S. Evangelou, D.J.N. Limebeer, R.S. Sharp, M.C. Smith, Control of motorcycle steering instabilities, IEEE Control Systems Magazine 26 (5) (2006) 78-88.

[14] S. Evangelou, D.J.N. Limebeer, R.S. Sharp, M.C. Smith, Mechanical steering compensators for high-performance motorcycles, Journal of Applied Mechanics 74 (2) (2007) $332-336$.

[15] F.C. Wang, M.K. Liao, B.H. Liao, W.J. Sue, H.A. Chan, The performance improvements of train suspension systems with mechanical networks employing inerters, Vehicle System Dynamics 47 (7) (2009) 805-830. 
[16] F.C. Wang, M.K. Liao, The lateral stability of train suspension systems employing inerters, Vehicle System Dynamics 48 (5) (2010) 619-643.

[17] F.C. Wang, M.R. Hsieh, H.J. Chen, Stability and performance analysis of a full-train system with inerters, Vehicle System Dynamics 50 (4) (2011) 545-571.

[18] J.Z. Jiang, A.Z. Matamoros-Sanchez, R.M. Goodall, M. C. Smith, Passive suspensions incorporating inerters for railway vehicles, Vehicle System Dynamics 50 (sup1) (2012) 263-276.

[19] F.C. Wang, M.F. Hong, C.W. Chen, Building suspensions with inerters, Proceedings of the Institution of Mechanical Engineers, Part C: Journal of Mechanical Engineering Science 224 (8) (2010) 1605-1616.

[20] W.T. Thomson, Theory of Vibration with Applications, 4th edition, Englewood Cliffs, NJ: Prentice-Hall, 1993.

[21] F.S. Tse, I.E. Morse, R.T. Hinkle, Mechanical vibrations, Maruzen Asian Edition, Allyn and Bacon, 1979.

[22] S.P. Beeby, M.J. Tudor, N.M. White, Energy harvesting vibration sources for microsystems applications, Measurement Science and Technology 17 (12) (2006) R175-R195.

[23] Y. Yu, N.G. Naganathan, R.V. Dukkipati, A literature review of automotive vehilce engine mounting systems, Mechanism and Machine Theory 36 (1) (2001) 123-142.

[24] R.A. Horn, C.R. Johnson, Matrix Analysis, New York: Cambridge Univ. Press, 1988.

[25] J.M. Kelly, G. Leitmann, A.G. Soldatos, Robust control of base-isolated structures under earthquake excitation, Journal of Optimization Theory and Applications 53 (1987) 159180.

[26] J.C. Ramallo, E.A. Johnson, B.F. Spencer, Smart base isolation systems, Journal of Engineering Mechanics (ASCE) 128 (10) (2002) 1088-1099.

[27] J. Zhao, J.T. DeWolf, Sensitivity study for vibrational parameters used in damage detection, Journal of structural engineering 125 (4) (1999) 410-416.

[28] J. Lin, R.G. Parker, Sensitivity of planetary gear natural frequencies and vibration modes to model parameters, Journal of Sound and Vibration 228 (1) (1999) 109-128.

[29] I.W. Lee, D.O. Kim, Natural frequency and mode shape sensitivities of damped systems: Part I, distinct natural frequencies, Journal of Sound and Vibration 223 (3) (1999) 399412. 


\section{Figure captions}

Figure 1: SDOF system with an inerter.

Figure 2: TDOF system with two inerters.

Figure 3: MDOF system with inerters.

Figure 4: The natural frequencies of the TDOF system. (a) $k_{2}>\left(1+m_{2} / m_{1}\right) k_{1}$; (b) $\left(1+m_{2} /\left(2 m_{1}\right)\right) k_{1} \leq k_{2} \leq\left(1+m_{2} / m_{1}\right) k_{1} ;$ (c) $k_{1} \leq k_{2}<\left(1+m_{2} /\left(2 m_{1}\right)\right) k_{1} ;$ (d) $k_{2} \leq k_{1}$. The red solid line: $\omega_{n 1}$; the blue dashed line: $\omega_{n 1}^{\prime}$; the red dash-dot line: $\omega_{n 2}$; the blue dotted line: $\omega_{n 2}^{\prime}$.

Figure 5: The permutation of natural frequencies of a three-degree-of-freedom system with $m_{i}=100 \mathrm{~kg}, k_{i}=1000 \mathrm{~N} / \mathrm{m}, i=1,2,3$ and $b_{1}=b_{3}=0 \mathrm{~kg}, b_{2} \in[0,600] \mathrm{kg}$.

Figure 6: The largest natural frequency of a six-degree-of-freedom system.

Figure 7: Procedures. (a) First step; (b) Second step: $b_{4}=5000 \mathrm{~kg}$; (c) Third step: $b_{4}=5000 \mathrm{~kg}, b_{2}=5000 \mathrm{~kg} ;(\mathrm{d})$ Fourth step: $b_{4}=5000 \mathrm{~kg}, b_{2}=5000 \mathrm{~kg}, b_{5}=5000 \mathrm{~kg} ;$ (e) Fifth step: $b_{4}=5000 \mathrm{~kg}, b_{2}=5000 \mathrm{~kg}, b_{5}=5000 \mathrm{~kg}, b_{3}=3000 ;$ (f) Sixth step: $b_{4}=5000$ $\mathrm{kg}, b_{2}=5000 \mathrm{~kg}, b_{5}=5000 \mathrm{~kg}, b_{3}=3000, b_{1}=1000 \mathrm{~kg}$. 


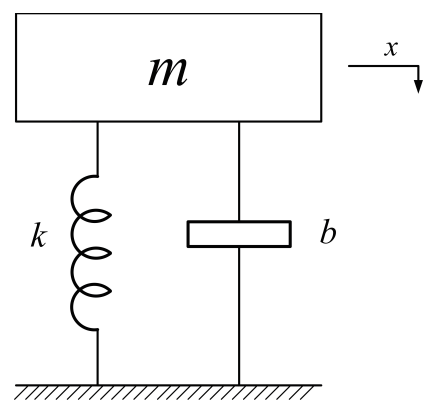

Figure 1: SDOF system with an inerter.

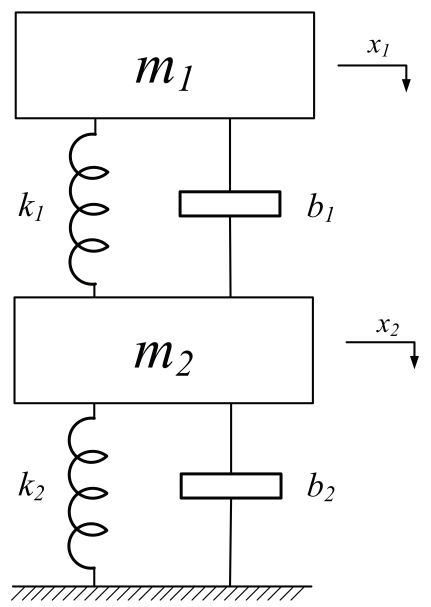

Figure 2: TDOF system with two inerters.

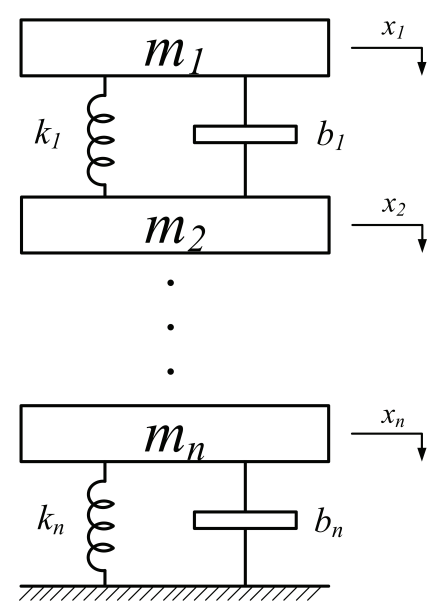

Figure 3: MDOF system with inerters. 


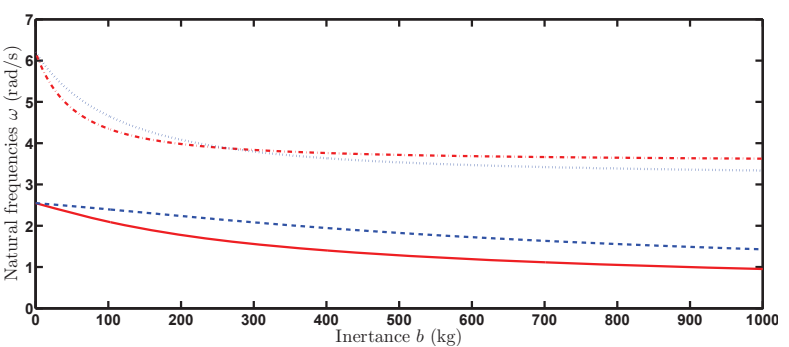

(a)

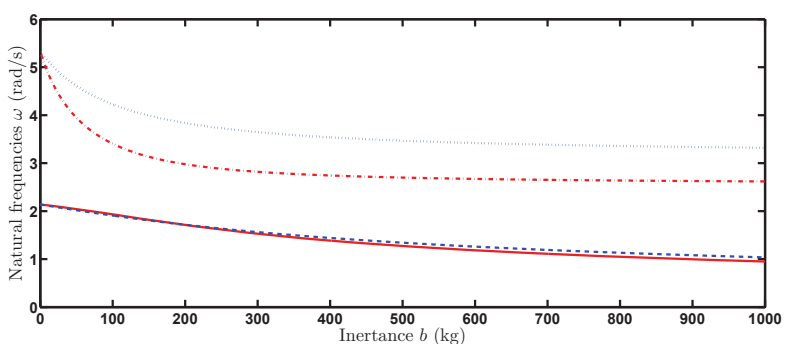

(c)

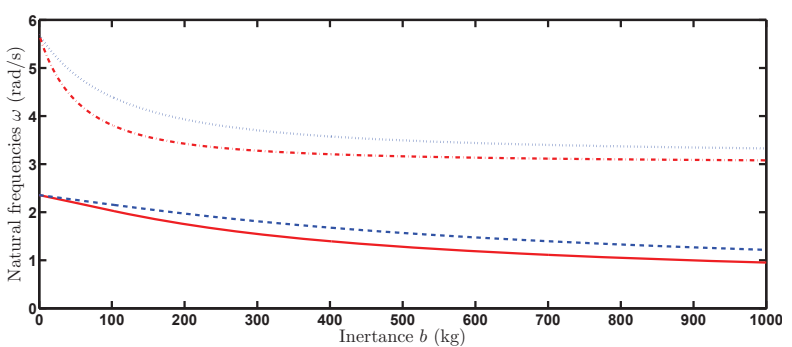

(b)

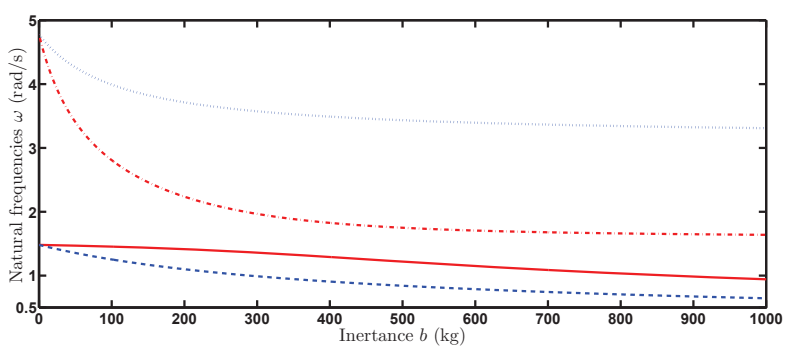

(d)

Figure 4: The natural frequencies of the TDOF system. (a) $k_{2}>\left(1+m_{2} / m_{1}\right) k_{1}$; (b) $\left(1+m_{2} /\left(2 m_{1}\right)\right) k_{1} \leq$ $k_{2} \leq\left(1+m_{2} / m_{1}\right) k_{1}$; (c) $k_{1} \leq k_{2}<\left(1+m_{2} /\left(2 m_{1}\right)\right) k_{1}$; (d) $k_{2} \leq k_{1}$. The red solid line: $\omega_{n 1}$; the blue dashed line: $\omega_{n 1}^{\prime}$; the red dash-dot line: $\omega_{n 2}$; the blue dotted line: $\omega_{n 2}^{\prime}$.

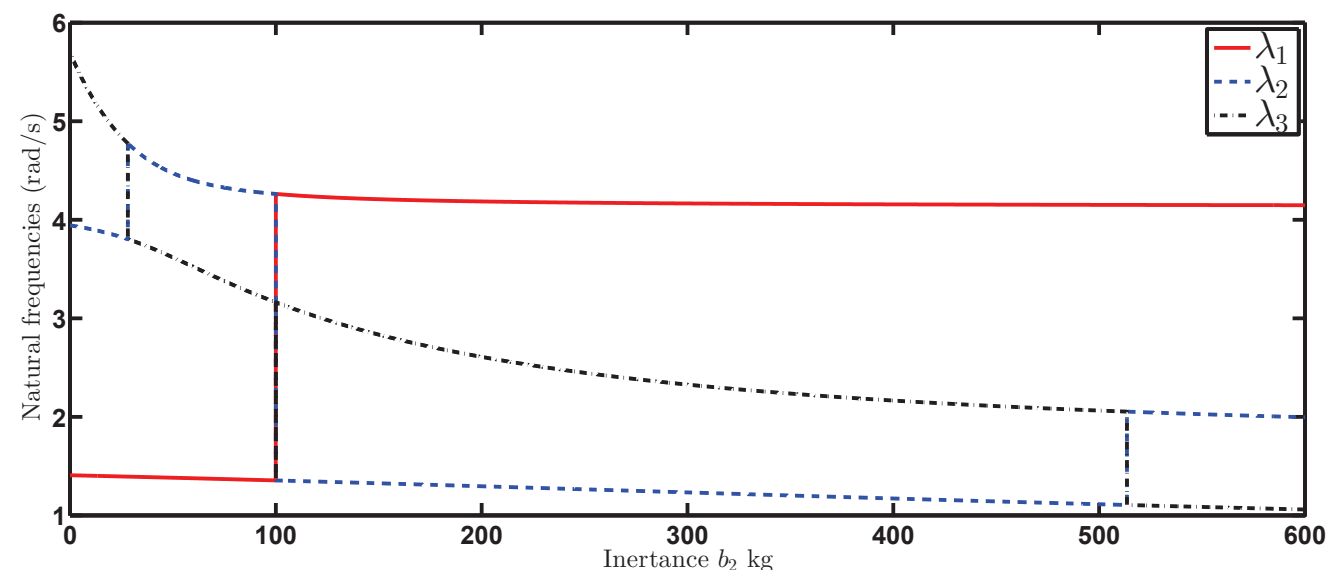

Figure 5: The permutation of natural frequencies of a three-degree-of-freedom system with $m_{i}=100 \mathrm{~kg}$, $k_{i}=1000 \mathrm{~N} / \mathrm{m}, i=1,2,3$ and $b_{1}=b_{3}=0 \mathrm{~kg}, b_{2} \in[0,600] \mathrm{kg}$. 


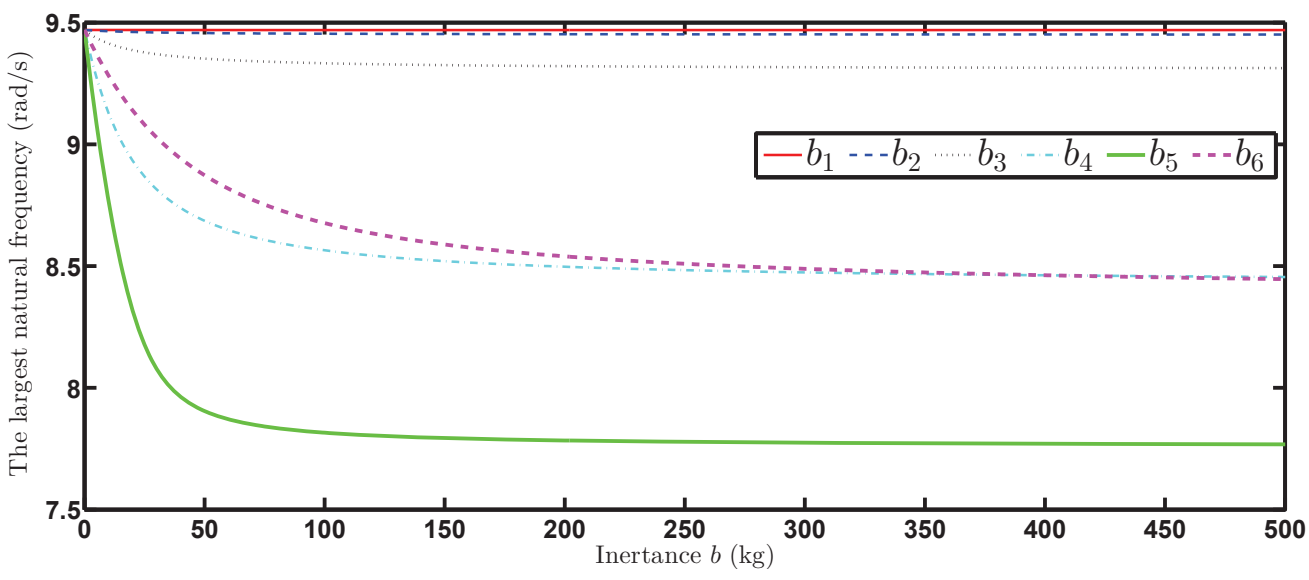

Figure 6: The largest natural frequency of a six-degree-of-freedom system. 


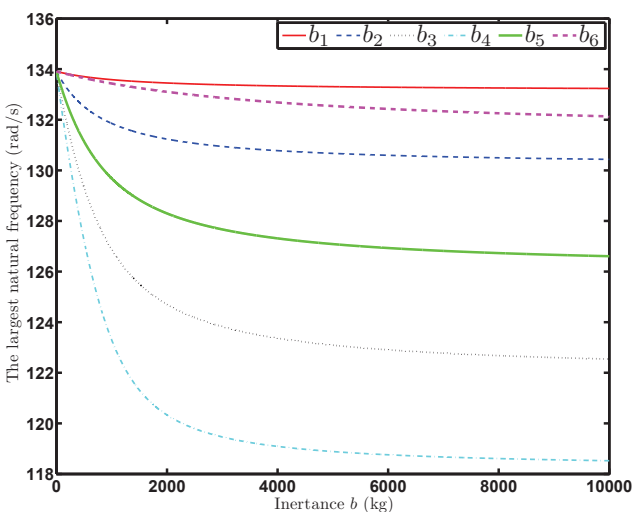

(a) First step

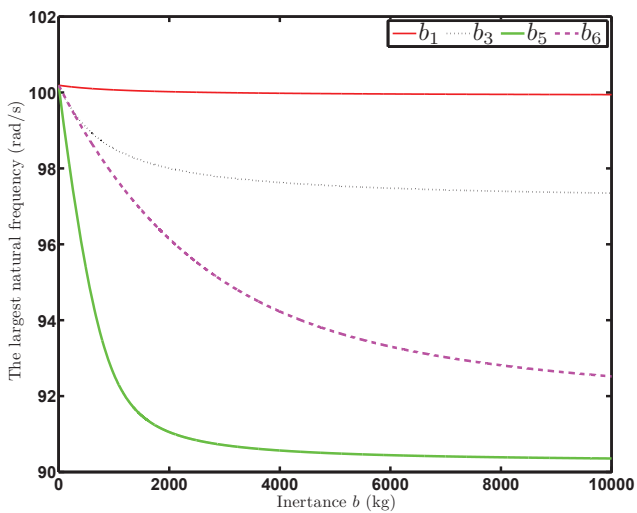

(c) Third step

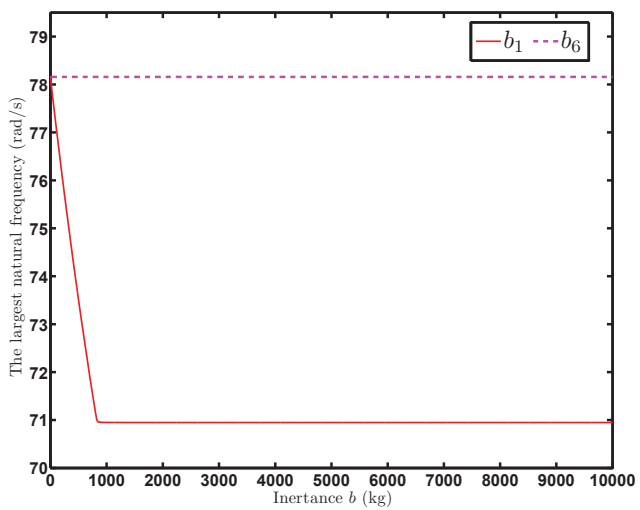

(e) Fifth step

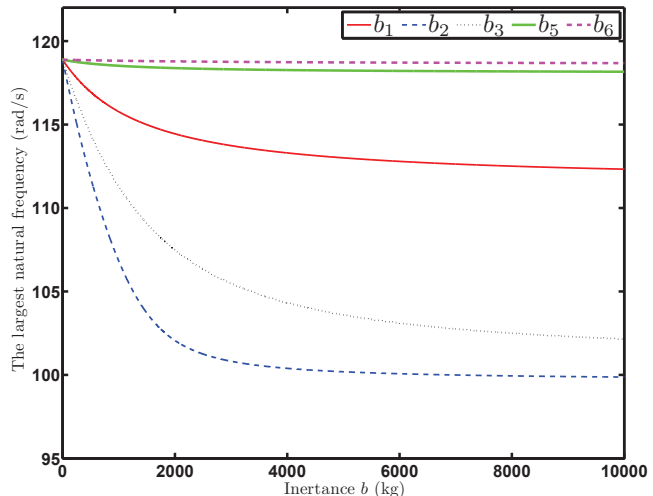

(b) Second step

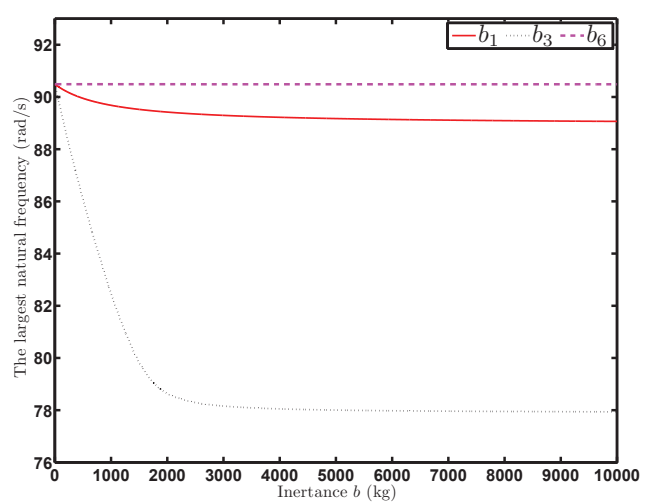

(d) Fourth step

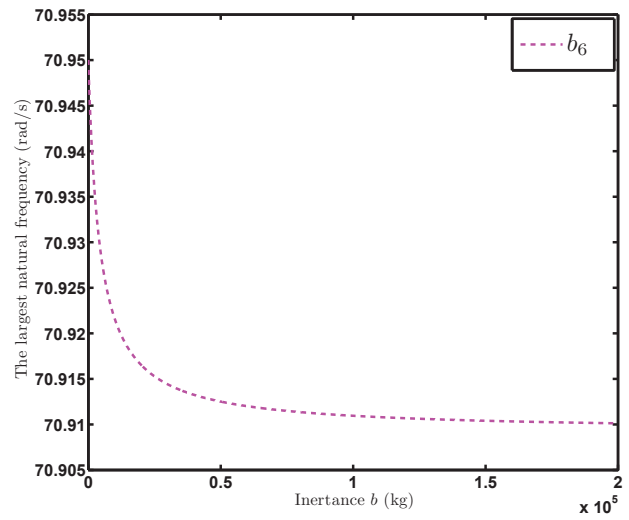

(f) Sixth step

Figure 7: Procedures. (a) First step; (b) second step: $b_{4}=5000 \mathrm{~kg}$; (c) third step: $b_{4}=5000 \mathrm{~kg}, b_{2}=5000$ $\mathrm{kg}$; (d) fourth step: $b_{4}=5000 \mathrm{~kg}, b_{2}=5000 \mathrm{~kg}, b_{5}=5000 \mathrm{~kg}$; (e) fifth step: $b_{4}=5000 \mathrm{~kg}, b_{2}=5000 \mathrm{~kg}$, $b_{5}=5000 \mathrm{~kg}, b_{3}=3000 \mathrm{~kg} ;$ (f) sixth step: $b_{4}=5000 \mathrm{~kg}, b_{2}=5000 \mathrm{~kg}, b_{5}=5000 \mathrm{~kg}, b_{3}=3000 \mathrm{~kg}, b_{1}=1000$ $\mathrm{kg}$. 